\title{
Contato "Pele a Pele" na Prevenção de Dor em Bebês Prematuros: Revisão Sistemática da Literatura
}

\author{
Camila Regina Lotto ${ }^{1}$ \\ Orcid.org/0000-0003-3914-2439 \\ Maria Beatriz Martins Linhares*, 1 \\ Orcid.org/0000-0001-5958-9874
}

${ }^{1}$ Universidade de São Paulo, São Paulo, SP, Brasil

\section{Resumo}

O presente estudo trata-se de uma revisão sistemática da literatura que teve por objetivo analisar a produção científica nacional e internacional sobre a efetividade do contato pele a pele inserido no Método Canguru como manejo preventivo da dor em bebês nascidos prematuros. O levantamento bibliográfico foi realizado nas bases PubMed, LILACS, SCielo e PsycINFO no período entre 2010 e 2017, por meio dos descritores kangaroo mother care method, skin-to-skin, pain e infant/newborn. Foram selecionados 12 artigos, que constituíram o corpus da revisão. Os resultados demonstraram a efetividade do contato pele a pele como técnica para alívio de dor aguda em bebês nascidos prematuros. O tempo de permanência na posição Canguru que ocasionou maior efetividade para o alívio de dor é o de 30 minutos antes do procedimento doloroso de rotina na Unidade de Tratamento Intensivo Neonatal. Além disso, a forma mais eficaz para realizar a avaliação da dor foi por meio da observação sistemática de indicadores comportamentais e fisiológicos, destacando-se a importância da utilização de instrumentos psicométricos validados e sensíveis para a dor em bebês prematuros. Ressalta-se também que o contato pele a pele pode trazer outros benefícios, como a promoção e fortalecimento do vínculo mãe-bebê e aleitamento materno.

Palavras-chave: Método canguru, pele a pele, dor, prematuro.

\section{"Skin-to-Skin" Contact in Pain Prevention in Preterm Infants: Systematic Review of Literature}

\begin{abstract}
The present study is a systematic review of the literature that aimed to analyze the national and international scientific production on the effectiveness of skin-to-skin contact included in the Kangaroo Method for preventive pain management in preterm infants. The literature review was performed in the PubMed, LILACS, SciELO and PsycINFO databases covering the period between 2010 and 2017, using the descriptors kangaroo mother care method, skin-to-skin, pain and infant/newborn. The search returned 12 articles, which constituted the corpus of the review. The results demonstrated the effecti-

* Endereço para correspondência: Universidade de São Paulo, Faculdade de Medicina de Ribeirão Preto, Rua Tenente Catão Roxo, 2650, Vila Monte Alegre, Ribeirão Preto, SP, Brasil 14051140. Fone: (16) 3315-4610; Fax: (16) 3315-4504 E-mail: linhares@fmrp.usp.br
\end{abstract}


veness of skin-to-skin contact as an acute pain relief technique in preterm infants. The time staying in the Kangaroo position that was more effective for pain relief was 30 minutes prior to the routine painful procedure in the Neonatal Intensive Care Unit. In addition, the most effective way to assess pain was through the systematic observation of behavioral and physiological indicators, highlighting the importance of the use of validated and sensitive psychometric instruments for pain in preterm infants. It was also emphasized that skin-to-skin contact can provide other benefits, such as promoting and strengthening the mother-baby attachment and encouraging breastfeeding.

Keywords: Kangaroo mother care method, skin-to-skin, pain, premature.

\section{Contacto "Piel a Piel” en la Prevención del Dolor en Bebés Prema- turos: Revisión Sistematica de la Literatura}

\section{Resumen}

El presente estudio se trata de una revisión sistemática de la literatura que tuvo por objetivo analizar la producción científica nacional e internacional sobre la efectividad del contacto piel a piel insertado en el Método Canguru como una gestión preventiva del dolor en bebés nacidos prematuros. El levantamiento bibliográfico fue realizado en las bases PubMed, LILACS, SCielo y PsycINFO en el período entre 2010 y 2017, por medio de los descriptores kangaroo mother care method, skin-to-skin, pain y infant/newborn. Se seleccionaron 12 artículos, que constituyen el corpus de la revisión. Los resultados demostraron la eficacia del contacto piel con piel como una técnica para el alivio del dolor agudo en los bebés prematuros. El tiempo de residencia en la posición canguro que causó más eficaz para el alivio del dolor es de 30 minutos antes del procedimiento doloroso de rutina en la Unidad de Cuidados Intensivos Neonatales. Además, la forma más eficaz para realizar la evaluación del dolor fue por medio de la observación sistemática de indicadores fisiológicos y del comportamiento, destacándose la importancia de la utilización de instrumentos psicométricos validados y sensibles para el dolor en bebés prematuros. Se resalta también que el contacto piel a piel puede traer otros beneficios, como la promoción y fortalecimiento del vínculo madre-bebé y lactancia materna.

Palabras clave: Método canguro, piel a piel, dolor, prematuro.

Os bebês nascidos prematuros $(<37$ semanas de idade gestacional [IG]), podem ser: extremo prematuro $(<28$ sem), muito prematuro $(28$ a $<32 \mathrm{sem})$, prematuro moderado (32 a $33 \mathrm{sem})$ e prematuro tardio (34 a 36 sem; Cassiano, Gaspardo, \& Linhares, 2016; Chabra, 2013; World Health Organization [WHO], 2016). A estimativa é que anualmente nascem 15 milhões de bebês pré-termo no mundo, portanto, sendo mais do que um bebê em cada 10 nascidos; a prematuridade é uma das principais causas de morte de crianças abaixo de cinco anos de idade (WHO, 2016). De acordo os dados do Sistema Único de Saúde (SUS), referentes ao ano de 2015, o índice de nascimentos prematuros no Brasil foi de $10,83 \%$, em um total de 3.017 .668 nascidos vivos (Ministério da Saúde, 2015).
A Unidade de Terapia Intensiva Neonatal (UTIN) assegura a proteção para sobrevivência dos neonatos pré-termo, entretanto, também os expõe a diversos procedimentos médicos, permeados por estímulos dolorosos e estressantes (Klein, Gaspardo, \& Linhares, 2006). Bebês internados na UTIN durante três meses tiveram 3.283 procedimentos invasivos, sendo mais frequentes a punção do calcâneo para coleta de sangue, a aspiração endotraqueal e a inserção intravenosa da cânula (Barker \& Rutter, 1995). Além disso, considerando-se o total de manipulações que os bebês sofrem na UTIN, verificou-se um total de 768 manipulações e 1.341 procedimentos, sendo que cada manipulação teve em média 2,2 procedimentos associados (Pereira et al., 2013). 
De acordo com a Associação Internacional para o Estudo da Dor (IASP, 1994/2017), a dor pode ser definida como: "uma experiência emocional e sensorial subjetiva desagradável associada a um dano real ou potencial de lesão tecidual, ou descrito em termos de tal dano" (IASP, 1994/2017, IASP Taxonomy para.3). O estudo de revisão sistemática de Valeri, Holsti e Linhares (2015) mostrou que o maior número de procedimentos estressores e dolorosos na fase neonatal associou-se a diversos impactos futuros, tais como: atrasos no crescimento após o nascimento e prejuízos no desenvolvimento motor e cognitivo. A dor neonatal acarreta a repetição do estímulo nocioceptivo que pode levar a respostas exageradas por parte do bebê e estas podem permanecer mesmo após o estímulo doloroso cessar (Grunau, 2002).

A inclusão da avaliação e do manejo da dor nos protocolos de cuidados intensivos ao bebê torna-se necessária para a proteção ao seu desenvolvimento (Linhares, 2016). Desta forma, é possível oferecer estratégias de prevenção ou alívio ao impacto dos estímulos nocioceptivos na fase neonatal, por meio de manejo farmacológico ou não farmacológico da dor (Linhares \& Doca, 2010). Entre as estratégias não farmacológicas destacam-se pela sua efetividade a sucção não-nutritiva, a sacarose (substância adocicada manipulada) via oral, o aleitamento materno, o toque palmar, o contato pele a pele, música, enrolamento do bebê, posicionamento e a estimulação olfatória e multissensorial (Cignacco et al., 2007; Linhares \& Doca, 2010). Especificamente o contato pele a pele, que associa-se ao Método Canguru ou Cuidado Mãe Canguru, tem sido uma das propostas para alívio de dor, podendo ser definido como uma intervenção naturalística que pode envolver a posição Canguru, a amamentação e o contato ao corpo materno (Warnock et al., 2010). O contato pele a pele para alívio de dor é preconizado no manual à Saúde do Recém-Nascido do Ministério da Saúde do Brasil como intervenção a ser implementada no contexto hospitalar (Ministério da Saúde, 2011). Segundo Charpak et al. (2005), o Método Canguru é uma intervenção considerada viável, disponível e preferida para diminuir a taxa de morbidade e mortalidade neonatal. Como este método inclui o contato pele a pele entre a mãe e o bebê, pode acarretar diversos impactos positivos para o bebê, tais como o controle fisiológico, controle da temperatura corporal, regulação do estado comportamental, incentivo ao vínculo mãe-bebê, incentivo à amamentação, entre outros. Em um estudo de meta-análise (Johnston et al., 2014) foram apresentadas evidências de como o contato pele a pele é efetivo para alívio de dor, demonstrando a capacidade de o bebê transitar suavemente entre o sono e vigília, mostrando que essa intervenção facilita o aumento da frequência do sono tranquilo dos bebês.

Estudos de revisão anteriores mostra-ram resultados positivos do contato pele a pele para alívio de dor em bebês nascidos pré-termo e a termo, analisando estudos pu-blicados de 2000 a 2009 (Warnock et al., 2010) e até janeiro de 2013 (Johnston et al., 2014). Verificou-se que os estudos de revisão anteriores não foram exclusivos sobre bebês nascidos pré-termo. Portanto, o objetivo do presente estudo foi revisar sistematica-mente os estudos publicados de 2010 a 2017, com o foco no manejo preventivo e de alívio da dor exclusivamente em bebês nascidos pré-termo, por meio da estratégia do contato pele a pele inserido no método Canguru utilizada durante a internação hospitalar na fase neonatal. A revisão visa atender às seguintes questões: (a) Quais as principais características dos estudos (país, delineamentos, tipo de amostra, escalas de medida de dor, variáveis mensuradas e contexto de dor)? (b) Quais os principais resultados sobre a efetividade do contato pele a pele inserido no método Canguru para alívio de dor em neonatos pré-termo?

\section{Método}

Procedeu-se a uma revisão sistemática de literatura científica como estratégia metodológica para se alcançar o objetivo proposto no presente estudo, seguindo os procedimentos propostos pelo Preferred Reporting Items for Systematic Reviews and Meta-Analyses - PRISMA (Liberati et al., 2009). A presente revisão bibliográfica teve como base a pesquisa de artigos empíricos 
publicados na base de dados PubMed, SciELO, LILACS e PsycINFO, com o recorte temporal de 2010 a 2017, adotado para avançar em relação aos estudos de revisão anteriores. Ressalta-se que em cada base, atentou-se para as variações necessárias, em função das especificidades de pesquisa de cada uma para localização dos estudos. Os descritores e palavras-chaves utilizados na busca dos artigos, de forma geral, foram os mesmos para todas as bases pesquisadas: kangaroo mother care method; skin-to-skin; pain; infant/newborn. Além disso, foram utilizados determinados filtros na base PubMed, sendo estes: somente publicações de 2010 a 2017 e pesquisas somente com seres humanos. Nas bases de dados LILACS, SciELO e PsycINFO, não foram utilizados filtros. Os critérios de inclusão estabelecidos foram os seguintes: (a) estudos empíricos que avaliaram o contato pele a pele ou método Canguru como estratégia de manejo de dor em bebês nascidos pré-termo; (b) artigos publicados nas línguas inglesa, portuguesa ou espanhola; (c) artigos publicados dentro do recorte temporal delimitado; (d) artigos referentes somente a seres humanos. Os critérios de exclusão, por sua vez foram os seguintes: (a) artigos de revisão,

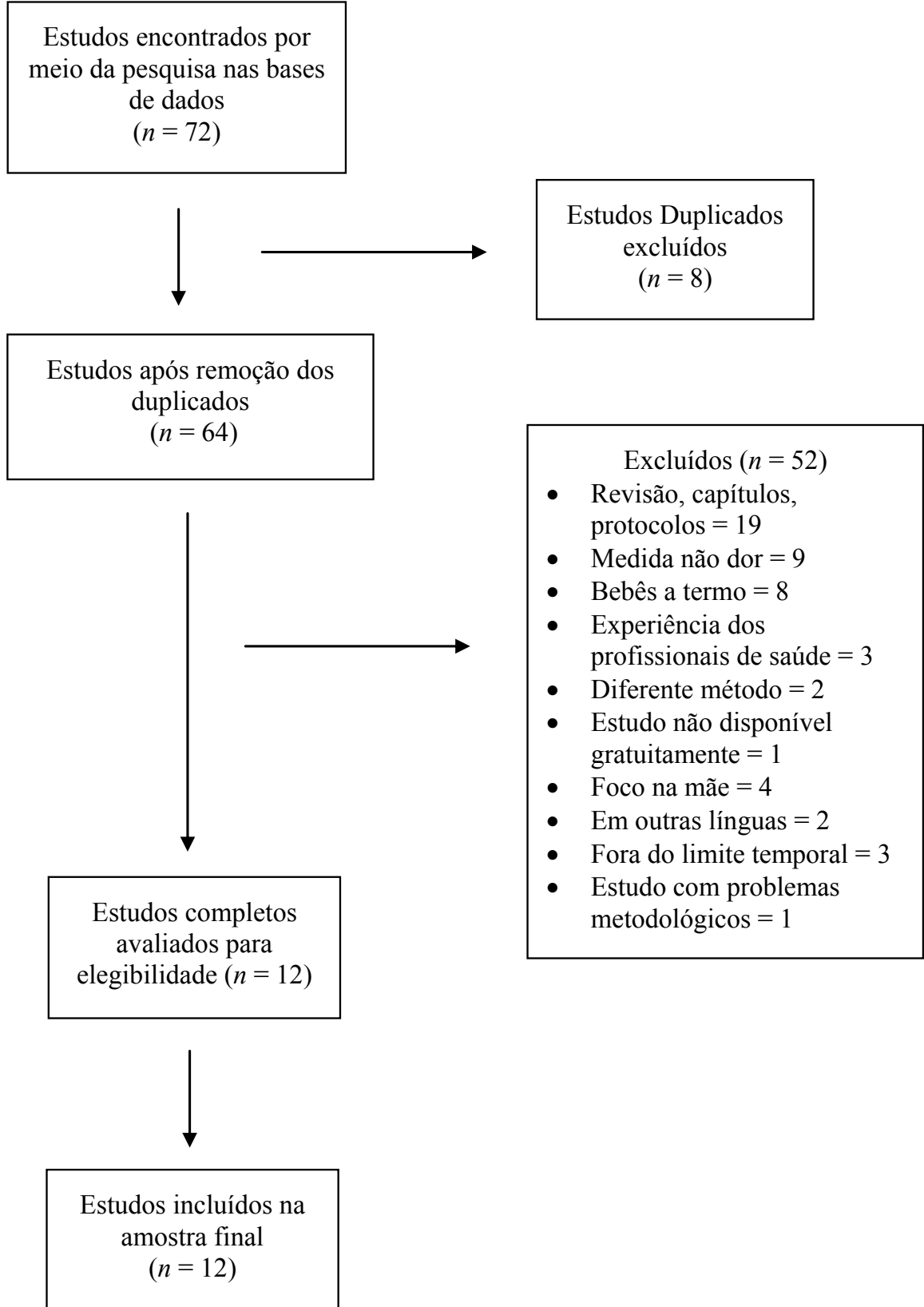

Figura 1. Fluxograma do processo de seleção dos estudos. 
meta-análise, comentários, protocolos de estudos, editoriais, cartas, capítulos de livros, dissertação, tese, manual, monografia ou crítica; (b) estudos com o foco nos profissionais de saúde e não nos bebês.

Verifica-se na Figura 1 que na busca realizada em abril de 2017, encontrou-se o total de 72 estudos. Em um primeiro momento, foram excluídos oito artigos por serem repetidos encontrados nas diferentes bases de dados (PubMed, LILACS e PsycINFO), restando 64 estudos. Em seguida, foi realizada a leitura dos títulos e resumos dos estudos, atentando-se para os objetivos propostos para esta revisão e aplicados os critérios de inclusão e exclusão por duas pesquisadoras, independentemente, e em caso de discordância as autoras discutiram até chegar a uma conclusão por consenso. Foram eliminados 52 artigos, pelos seguintes motivos: (a) artigos de revisão, capítulos de livro, protocolos de estudos, protocolos de método $(n=19)$; (b) não utilizou a dor como medida $(n=9)$; (c) a amostra incluía apenas bebês nascidos a termo $(\mathrm{n}=8)$; (d) o estudo avaliava a experiência dos profissionais de saúde $(n=3)$; (e) o estudo utilizava outro método para aliviar a dor, farmacológico ou não farmacológico $(n=2)$; (f) o artigo não estava disponível gratuitamente $(n=1) ;(\mathrm{g})$ o foco do estudo era somente na mãe, sem a medida da dor $(n=4)$; (h) artigos em outras línguas $(n=2)$; (i) artigos encontrados fora do limite temporal delimitado $(n=3)$; artigo com problemas metodológicos que invalidavam os resultados $(n=1)$. Foram selecionados 12 estudos para a avaliação de elegibilidade e a amostra final foi composta por 12 estudos.

Os artigos selecionados foram revisados e analisados de acordo com os seguintes tópicos: objetivo estabelecido pelo estudo; amostra; delineamento do estudo; intervenção; variáveis observadas; escalas de mensuração de dor; principais resultados do estudo; limitações da pesquisa. Os artigos foram lidos na íntegra e fichados de acordo com esses itens pela primeira autora e, posteriormente, foram revisados pela segunda autora, de forma independente, a fim de assegurar a precisão das análises.

\section{Resultados}

\section{Características Gerais dos Estudos}

Primeiramente, serão apresentadas as características dos estudos que se encontram resumidas na Tabela 1 .

Os 12 artigos revisados foram publicados predominantemente no ano de $2012(\mathrm{n}=4,34 \%)$ e os demais distribuíram-se entre $2013(\mathrm{n}=3$, 25\%), $2011(\mathrm{n}=2,17 \%), 2014(\mathrm{n}=1,8 \%) 2015$ $(\mathrm{n}=1,8 \%)$ e $2016(\mathrm{n}=1,8 \%)$. A maioria dos estudos foram conduzidos em países da América do Norte ( $\mathrm{n}=4$, nos EUA, e $\mathrm{n}=2$, no Canadá), seguindo-se da Ásia ( $\mathrm{n}=3$, na Índia, e $\mathrm{n}=1$, China), apenas um da América do Sul (no Brasil) e um da Europa (Suécia).

Os delineamentos adotados nos estudos foram os seguintes: em $41 \%$ dos estudos, estudo clínico randomizado cruzado (Cong, Cusson, Walsh, et al., 2012; Cong et al., 2011; Johnston et al., 2012; Johnston et al., 2011; Nimbalkar et al., 2013), em 25\% dos estudos, estudo clínico randomizado controlado (Gao et al., 2015; Mitchell et al., 2013; Nanavati et al., 2013) e 17\% dos estudos, estudo clínico cruzado (Chidambaram et al., 2014; Olsson et al., 2016). Os demais estudos (17\%) foram estudo de caso, descritivo e exploratório (Cong, Cusson, Hussain, et al., 2012) e outro estudo descritivo, exploratório e correlacional (Castral et al., 2012).

Quanto à amostra estudada, de forma geral, observou-se que a IG dos bebês foi um critério de inclusão importante para os estudos analisados, sendo que esta variou de 27 a 37 semanas. Um estudo utilizou bebês de extremamente prematuros a muito prematuros (Mitchell et al., 2013), três estudos, bebês muito prematuros (Cong, Cusson, Hussain, et al., 2012; Cong, Cusson, Walsh, et al., 2012; Cong et al., 2011), dois estudos, bebês de moderada a tardia prematuridade (Chidambaram et al., 2014; Nimbalkar et al., 2013) e cinco utilizaram uma classificação geral dos bebês, de 28 a 36 semanas (Castral et al., 2012; Gao et al., 2015; Johnston et al., 2012; Johnston et al., 2011; Olsson et al., 2016). Destaca-se que somente um estudo realizou uma média com as idades gestacionais, obtendo uma 
Tabela 1

Síntese da Metodologia dos Estudos $(n=12)$

\begin{tabular}{|c|c|c|c|c|c|c|}
\hline Autor/Ano & Delineamento & Amostra & $\begin{array}{l}\text { Procedi- } \\
\text { mento } \\
\text { de dor }\end{array}$ & Intervenção & Variável & $\begin{array}{c}\text { Instrumento } \\
\text { avaliação } \\
\text { de dor }\end{array}$ \\
\hline (Castral et al., 2012) & $\begin{array}{l}\text { Edescr, expl } \\
\text { e correl }\end{array}$ & $\begin{array}{l}49 \text { RN PT, IG } \\
<36 \text { s e } 6 \text { d. }\end{array}$ & $\mathrm{PC}$ & $\mathrm{MC}$ & $\begin{array}{l}\text { Comp, } \\
\text { fisiol. e } \\
\text { saliva }\end{array}$ & NFCS \\
\hline $\begin{array}{l}\text { (Chidambaram, Manjula, } \\
\text { Adhisivam, \& Bhat, 2014) }\end{array}$ & $\mathrm{ECC}$ & $\begin{array}{l}50 \mathrm{RN}, \mathrm{PT} \\
\mathrm{IG} 32-36 \mathrm{~s} \\
\mathrm{PN}<2.500 \mathrm{~g}\end{array}$ & $\mathrm{PC}$ & MC X Ic & $\begin{array}{l}\text { Comp e } \\
\text { fisiol }\end{array}$ & PIPP \\
\hline $\begin{array}{l}\text { (Cong, Cusson, Hussain, Zhang, } \\
\text { \& Kelly, 2012) }\end{array}$ & $\mathrm{EC}$ & $\begin{array}{l}\text { Gêmeos, IG } \\
28 \mathrm{~s}\end{array}$ & $\mathrm{PC}$ & $\begin{array}{l}\text { MC (30min e } \\
15 \text { min) X Ic }\end{array}$ & $\begin{array}{l}\text { Comp e } \\
\text { fisiol }\end{array}$ & $\begin{array}{l}\text { PIPP e } \\
\text { ABSS }\end{array}$ \\
\hline (Cong, Cusson, Walsh, et al., 2012) & ERC & $\begin{array}{l}26 \mathrm{RN} \text { PT, IG } \\
28-32 \mathrm{~s}\end{array}$ & $\mathrm{PC}$ & $\begin{array}{l}\mathrm{MC}(30 \mathrm{~min} \mathrm{e} \\
15 \mathrm{~min}) \mathrm{X} \mathrm{Ic}\end{array}$ & $\begin{array}{l}\text { Comp e } \\
\text { fisiol }\end{array}$ & ABSS \\
\hline $\begin{array}{l}\text { (Cong, Ludington-Hoe, \& Walsh, } \\
\text { 2011) }\end{array}$ & ERC & $\begin{array}{l}18 \text { RN PT, IG } \\
\text { de } 30-32 \mathrm{~s}\end{array}$ & $\mathrm{PC}$ & $\begin{array}{l}\text { MC (80min e } \\
30 \mathrm{~min}) \mathrm{X} \mathrm{Ic}\end{array}$ & $\begin{array}{l}\text { Comp, } \\
\text { fisiol. E } \\
\text { saliva }\end{array}$ & PIPP \\
\hline (Gao et al., 2015) & ERCo & $\begin{array}{c}75 \mathrm{RN} \text { PT, IG } \\
\quad<37 \mathrm{~s}\end{array}$ & $\mathrm{PC}$ & MC X Ic & $\begin{array}{l}\text { Comp e } \\
\text { fisiol }\end{array}$ & $\begin{array}{l}\text { Não } \\
\text { utilizou }\end{array}$ \\
\hline (Johnston et al., 2012) & ERC & $\begin{array}{l}18 \text { RN PT, IG } \\
28-36 \mathrm{~s}\end{array}$ & $\mathrm{PC}$ & $\mathrm{MC}$ & $\begin{array}{l}\text { Comp e } \\
\text { fisiol }\end{array}$ & PIPP \\
\hline $\begin{array}{l}\text { (Johnston, Campbell-Yeo, \& Filion, } \\
\text { 2011) }\end{array}$ & ERC & $\begin{array}{l}62 \mathrm{RN} \text { PT, IG } \\
28-36 \mathrm{~s}\end{array}$ & $\mathrm{PC}$ & $\mathrm{MC}$ & $\begin{array}{l}\text { Comp e } \\
\text { fisiol }\end{array}$ & PIPP \\
\hline $\begin{array}{l}\text { (Mitchell, Yates, Williams, Chang, } \\
\text { \& Hall, 2013) }\end{array}$ & ERCo & $\begin{array}{l}38 \mathrm{RN} \text { PT, IG } \\
27-30 \mathrm{~s}\end{array}$ & $\begin{array}{l}\text { Aspiração } \\
\text { traqueal ou } \\
\text { nasal }\end{array}$ & MC X Ic & $\begin{array}{l}\text { Comp, } \\
\text { fisiol. E } \\
\text { saliva }\end{array}$ & PIPP \\
\hline (Nanavati, Balan, \& Kabra, 2013) & ERCo & $\begin{array}{c}1^{\mathrm{o}}: 15 \mathrm{RN}, \mathrm{PT}, \\
\text { IG } 32 \mathrm{~s} \\
2^{\mathrm{o}}: 50 \mathrm{RN} .\end{array}$ & $\begin{array}{l}\text { Remoção de } \\
\text { esparadrapo }\end{array}$ & MC X aleit. & $\begin{array}{l}\text { Comp e } \\
\text { fisiol }\end{array}$ & PIPP \\
\hline $\begin{array}{l}\text { (Nimbalkar, Chaudhary, Gadhavi, \& } \\
\text { Phatak, 2013) }\end{array}$ & ERC & $\begin{array}{c}47 \text { RN PT, } \\
\text { PN<2.500g, } \\
\text { IG 32-36s }\end{array}$ & $\mathrm{PC}$ & $\begin{array}{l}\mathrm{MC}(15 \mathrm{~min}) \\
\mathrm{X} \mathrm{Ic}\end{array}$ & $\begin{array}{l}\text { Comp e } \\
\text { fisiol }\end{array}$ & PIPP \\
\hline (Olsson, Ahlsén, \& Eriksson, 2016) & $\mathrm{ECC}$ & $\begin{array}{c}10 \mathrm{RN} \text { PT, IG } \\
26-35 \mathrm{~s}\end{array}$ & $\begin{array}{l}\text { Punção ve- } \\
\text { nosa }\end{array}$ & MC X Ic & $\begin{array}{l}\text { Comp, } \\
\text { fisiol e } \\
\text { NIRS. }\end{array}$ & PIPP \\
\hline
\end{tabular}

Nota. ERC - Estudo Clínico Randomizado Cruzado; ERCo - Estudo Clínico Randomizado Controlado; ECC - Estudo Clínico Cruzado; Edescr, expl e correl - Estudo Descritivo, Exploratório e Correlacional; EC - Estudo de Caso; PT - Pré-Termo; IG Idade Gestacional; PC - Punção do Calcâneo; s - Semanas; DPN - Dias Pós Natal; PN - Peso ao Nascimento; MC - Método Canguru; Ic - Incubadora; comp - Comportamento; fisiol. - Fisiológico (frequência cardíaca e saturação oxigênio); PIPP Premature Infant Pain Profile; ABSS - Anderson Behavioral State Scoring System; NFCS - Neonatal Facial Coding System; d - Dias; aleit - Aleitamento Materno; NIRS - Near-infrared Spectroscopy.

classificação de prematuro moderado (Nanavati et al., 2013).

Vale destacar os critérios de inclusão e exclusão das amostras dos estudos. De um lado, como critério de inclusão, os bebês incluídos em todos os estudos estavam clinicamente estáveis para poder participar da intervenção do contato pele a pele. Por outro lado, os principais critérios de exclusão foram os seguintes: bebês clinicamente instáveis, que passaram por cirurgias recentes, receberam sedativos ou analgésicos, com hemorragia intraventricular grave (graus III 
e IV), com anomalias congênitas, com más formações e/ou com danos neurológicos.

Em geral, os bebês nascidos pré-termo foram avaliados antes dos 15 dias de idade pósnatal, sendo: menos cinco dias (Mitchell et al., 2013), dois a nove dias (Cong et al., 2011); de 10 dias (Johnston et al., 2012; Nimbalkar et al., 2013) e 15 dias (Cong, Cusson, Walsh, et al., 2012; Gao et al., 2015). Os demais estudos não especificaram as idades de nascimento dos bebês. Destaca-se que somente em alguns estudos o peso ao nascimento dos bebês foi considerado como um critério de inclusão, sendo que em cinco destes foi especificado o peso dos bebês, menos de $2.500 \mathrm{~g}$ (Chidambaram et al., 2014; Nimbalkar et al., 2013) e menos de $1.500 \mathrm{~g}$ (Cong, Cusson, Hussain, et al., 2012; Mitchell et al., 2013; Nanavati et al., 2013).

Quanto à avaliação da dor dos bebês, na maioria dos estudos $(\mathrm{n}=10,83 \%)$, foram utilizados instrumentos psicométricos validados especificamente para a mensuração da dor, sendo que em nove estudos, utilizou-se a Premature Infant Pain Profile - PIPP, que é um instrumento multidimensional composto por indicadores comportamentais, fisiológicos e contextuais da ocorrência da dor específico para recém-nascidos pré-termo (Chidambaram et al., 2014; Cong, Cusson, Walsh, et al., 2012; Cong et al., 2011; Johnston et al., 2012; Johnston et al., 2011; Mitchell et al., 2013; Nanavati et al., 2013; Nimbalkar et al., 2013; Olsson et al., 2016) e apenas um estudo utilizou o Neonatal Facial Coding System - NFCS, instrumento em que tem como medida a atividade facial de bebês nascidos pré-termo ou a termo (Castral et al., 2012). No restante dos artigos, utilizouse um instrumento em que se mede o estado comportamental do bebê, em um contexto de procedimento doloroso, sendo este o Anderson Behavioral State Scoring System - ABSS, que é uma escala que tem por objetivo medir o estado comportamental dos bebês e possui 12 categorias $(1=$ sono muito calmo; $2=$ sono calmo; $3=$ sono ativo; $4=$ sono muito ativo; $5=$ sonolento; $6=$ inativo alerta; $7=$ acordado calmo; $8=$ acordado ativo; $9=$ acordado muito ativo; $10=$ choramingando; 11 = chorando; e $12=$ chorando muito; Cong, Cusson, Hussain, et al., 2012; Cong, Cusson, Walsh, et al., 2012), em um dos artigos a ABSS foi associada ao PIPP. Apenas um artigo não utilizou instrumento psicométrico validado para a identificação de dor, porém utilizou indicadores comportamentais de dor, por meio da duração da mímica facial e do choro (Gao et al., 2015).

No que se refere aos tipos de procedimentos em que a dor foi avaliada, primeiramente deve-se destacar que em todos os estudos os procedimentos ocorreram por demanda clínica. Verificou-se que em nove artigos a punção do calcâneo foi o procedimento de dor avaliado. Destaca-se que houve diversas nomeações para esse procedimento, sendo estas: "heel stick", "heel pricking", "capillary blood", "blood draws" e "heel lance procedure". Os demais artigos avaliaram a dor no procedimento de aspiração traqueal ou nasal (Mitchell et al., 2013), punção venosa (Olsson et al., 2016) ou remoção de esparadrapo (Nanavati et al., 2013).

Quanto às variáveis utilizadas para avaliar a dor, pode-se observar um certo padrão nos estudos analisados. De modo geral, o comportamento (atividades faciais, tempo de choro, mímica facial) e as medidas fisiológicas (frequência cardíaca, saturação de oxigênio e cortisol salivar) foram as variáveis utilizadas nos estudos. Dos 12 estudos selecionados, nove optaram por analisar em conjunto as medidas fisiológicas e os indicadores comportamentais (Chidambaram et al., 2014; Cong, Cusson, Hussain, et al., 2012; Cong, Cusson, Walsh, et al., 2012; Gao et al., 2015; Johnston et al., 2012; Johnston et al., 2011; Nanavati et al., 2013; Nimbalkar et al., 2013; Olsson et al., 2016). Quanto ao cortisol salivar, medida também fisiológica, somente três estudos optaram por incluí-la (Castral et al., 2012; Cong et al., 2011; Mitchell et al., 2013) em conjunto a outras medidas fisiológicas (frequência cardíaca e saturação de oxigênio) e indicadores comportamentais. Destaca-se que nestes estudos, além de se investigar a dor, analisou-se também o estresse causado pelos procedimentos de dor no prematuro. Somente um estudo utilizou-se também de um procedimento diferente para a avaliação da dor, em conjunto às medi- 
das citadas anteriormente (frequência cardíaca, saturação de oxigênio e comportamento), sendo este a Near-infrared spectroscopy (NIRS), uma técnica não invasiva, que reflete alterações hemodinâmicas em partes regionais do cérebro (Olsson et al., 2016). Assim, para se ter um olhar amplo acerca dos impactos dos procedimentos dolorosos para o bebê e como a intervenção atua na dor, a análise dessas medidas será realizada em conjunto com a maneira em que a técnica de intervenção do contato pele a pele foi abordada pelos estudos.

É importante ressaltar como o método do contato pele a pele foi praticado nos diferentes estudos. O bebê usava somente a fralda, era colocado no peito da mãe ou outro cuidador, na posição pele a pele e ambos cobertos por um pano ou cobertor, em uma posição inclinada de 30 a 40 graus. O tempo em que o bebê e a mãe permaneciam nesta posição variou de um estudo para estudo, podendo ser de 15 a 80 minutos. $\mathrm{Na}$ maioria dos estudos, não houve uma especificação quanto à interação da mãe/cuidador com o bebê durante a intervenção para alívio de dor, sendo que somente em um artigo houve o pedido para que a mãe evitasse qualquer tipo interação (Nimbalkar et al., 2013) e em dois, que especificaram que a mãe poderia interagir livremente com o bebê (Castral et al., 2012; Cong, Cusson, Hussain, et al., 2012).

\section{Principais Resultados sobre a Efetividade do Contato Pele a Pele Inserido no Método Canguru para Alívio de Dor em Neonatos Pré-Termo}

Os principais resultados dos estudos foram organizados em tópicos temáticos, que emergiram da análise dos artigos. Primeiramente, verificou-se um conjunto de estudos que compararam grupos de neonatos pré-termo submetidos ao contato pele a pele no Método Canguru para alívio de dor e grupos controle. Em segundo lugar, houve estudos cujo foco consistia na análise do efeito do tempo de exposição dos neonatos pré-termo ao contato pele a pele no Método Canguru durante o procedimento doloroso. Em terceiro lugar, notou-se que em determinados estudos o objetivo consistia em avaliar o efeito do contato pele a pele no Método Canguru realizado por diferentes cuidadores para alívio de dor. Em quarto lugar, apenas um estudo teve como foco a comparação do contato pele a pele no Método Canguru com a amamentação para alívio de dor. Finalmente, um estudo analisou a associação entre indicadores maternos e o contato pele a pele no Método Canguru para alívio de dor.

Método Canguru (Kangaroo Care) no alívio de dor vs. Cuidado padrão de rotina (Standard Care). O Método Canguru, em comparação com o bebê na incubadora, no alívio de dor foi realizado em cinco dos artigos selecionados. O estudo de Nimbalkar et al. (2013) comparou 47 bebês com peso ao nascimento menor do que $2.500 \mathrm{~g}$, entre 32 e 36 semanas de idade gestacional (IG) e com 10 dias pós-natal. Utilizou-se o Método Canguru para alívio da dor durante 30 minutos (15 minutos durante a punção de calcâneo e 15 minutos após este procedimento) em comparação ao cuidado padrão de rotina, em que o bebê permanecia na incubadora, sem intervenção para manejo de dor. Quanto às formas de avaliação da dor, utilizou-se da gravação em vídeo da face e do comportamento do bebê, além de gravar os monitores da frequência cardíaca e da saturação de oxigênio, desde a linha de base inicial até a fase de recuperação após o procedimento doloroso. Destaca-se que para medir a dor, o instrumento utilizado foi a Premature Infant Pain Profile (PIPP). Dessa forma, os bebês na posição Canguru obtiveram escores na PIPP significativamente menores do que os bebês que permaneceram na incubadora. Além disso, a frequência cardíaca dos bebês foi significativamente reduzida com o contato pele a pele.

De forma semelhante, Gao et al. (2015) mostram a efetividade do contato pele a pele por 30 minutos no alívio de dor dos bebês pré-termo durante a punção do calcâneo. A duração do choro do bebê, a mímica facial (fronte saliente, olhos apertados e o sulco nasolabial franzido) e a frequência cardíaca foram analisadas. Verificou-se uma convergência entre os resultados das medidas fisiológica e comportamental, mostrando que houve uma redução da frequência cardíaca, da duração do choro e da mímica facial 
durante o procedimento de dor em bebês que estavam na posição Canguru em comparação aos bebês na incubadora. No estudo de Olsson et al. (2016), o contato pele a pele produziu alívio da dor durante o procedimento de punção venosa. Um destaque deste estudo foram as medidas utilizadas para avaliar a dor, no sentido de utilizar, além das medidas fisiológicas (frequência cardíaca e saturação do oxigênio) e comportamentais, utilizou-se também a NIRS (técnica não invasiva que reflete alterações hemodinâmicas em partes regionais do cérebro). Este estudo analisou 10 bebês pré-termo usando a PIPP. Os resultados obtidos assemelham-se aos estudos anteriores, ao ter mais respostas fisiológicas à dor nos bebês que se encontravam na incubadora, do que os que estavam sob a intervenção. Os escores PIPP também foram mais elevados durante o cuidado padrão do que no contato pele a pele, entretanto não houve diferença significativa entre esses grupos. Seguindo nesta mesma linha, o estudo de Chidambaram et al. (2014) analisou 50 bebês pré-termo, com IG de moderada a tardia, pesando menos de $2.500 \mathrm{~g}$. Para analisar a dor, foram utilizadas medidas fisiológicas (frequência cardíaca e saturação do oxigênio) e a PIPP, sendo utilizada a escala 15 minutos antes do procedimento e 15 e 30 minutos após em ambos os grupos. Os escores da PIPP foram significativamente menores no grupo do Canguru em comparação ao controle.

Em contrapartida, no estudo de Mitchell et al. (2013) houve resultados divergentes. Neste estudo, 38 bebês com IG entre 27 e 30 semanas, com menos de cinco dias de idade pós-natal, foram testados dividindo-se em dois grupos: 19 bebês no grupo do contato pele a pele, foram colocados na posição Canguru por duas horas, diariamente e consecutivamente por cinco dias, e 19 bebês permaneceram sob cuidado padrão de rotina, porém a pedido dos pais, poderiam ser colocados na posição Canguru por 15 minutos diariamente. $\mathrm{Na}$ avaliação foi utilizada a medida do cortisol salivar e dos indicadores de dor por meio da PIPP. Observou-se uma diminuição no cortisol salivar comparando-se os dias de teste, sendo mais alto o nível de cortisol no primeiro dia do que no último, porém não houve uma mu- dança deste nível entre os grupos analisados (posição Canguru e posição na incubadora); quanto aos valores da PIPP, estes foram elevados após o procedimento de dor (aspiração traqueal ou nasal) em comparação com o momento inicial do estudo, sem o procedimento, permanecendo inalterados durante todos os dias de teste. Destaca-se que a intervenção para manejo do alívio da dor não foi realizada antes ou durante a aspiração.

Método Canguru com diferentes durações para o alívio da dor. Nesta categoria, três artigos, além de examinar a efetividade da posição Canguru no alívio da dor em bebês nascidos prematuros, investigaram também o tempo ideal para que o contato pele a pele seja mais eficaz. No estudo de Cong, Cusson, Hussain, et al. (2012), verificou-se a efetividade do método Canguru no estudo de caso de um casal de bebês gêmeos. Os bebês analisados tinham a IG de 28 semanas e eram de extremo baixo peso ao nascimento $(1.500 \mathrm{~g})$ e foram testados em três condições: o Canguru longo (30 minutos), o Canguru curto (15 minutos) e os cuidados padrões de rotina (bebês ficavam na incubadora) sem intervenção para alívio de dor. Na avaliação da dor, recorreu-se a medidas fisiológicas (frequência cardíaca e respiratória), o estado comportamental do bebê medido pela Anderson Behavior State Scoring System (ABSS) e a PIPP. Para medir a frequência cardíaca e respiratória do bebê foram utilizados eletrodos, colocados nos peitos dos bebês, acessando as respostas nervosas autonômicas do bebê (variabilidade da frequência cardíaca).

Assim, durante a punção do calcâneo, os bebês apresentaram menos sinais de dor na posição Canguru do que na incubadora. Os resultados desse estudo demonstraram que com a intervenção para alívio de dor, a razão entre esses extremos foi menor, o que indica que os efeitos do contato pele a pele inserido no Método Canguru são atenuantes nas respostas autonômicas da dor. Quanto aos indicadores comportamentais, observou-se pela ABSS, um tempo maior e regular em sono calmo durante o Canguru longo e o curto em comparação à condição da incubadora, que ficou por mais tempo em um sono 
ativo nesta última condição. Quanto aos escores da PIPP, ambos os bebês apresentaram valores mais elevados clinicamente durante a condição sem intervenção para alívio de dor, durante o procedimento, do que no Canguru, tanto curto quanto longo. Em comparação entre ambos os Cangurus, observou-se apenas uma tendência de os bebês apresentarem mais sinais de dor (o choro) durante o Canguru longo (30 minutos) do que o curto (15 minutos).

O estudo de Cong, Cusson, Walsh, et al. (2012), utilizou as mesmas durações de tempo (15 e 30 minutos) na posição Canguru do estudo anterior e a posição na incubadora, sem intervenção para manejo da dor. Neste estudo, foram observados 26 bebês, com IG de 28 a 32 semanas, com menos que 14 dias pós-natal. Os bebês passaram por todas as três condições explicitadas anteriormente, porém em ordens diferentes. Para a avaliação da dor, foram utilizadas as mesmas medidas fisiológicas do estudo anterior, a variabilidade da frequência cardíaca, em conjunto aos indicadores comportamentais apontados pelo estado do bebê (ABSS). Neste estudo não foi utilizada uma escala para medir a dor, mantendo-se um foco nas medidas fisiológicas e nas respostas autonômicas. Na fase de recuperação não houve diferença significativa entre os dois tempos na posição Canguru. Porém em comparação com a incubadora, verificou-se que a variabilidade da frequência cardíaca, foi significativamente menor na posição Canguru por 30 minutos.

Em outro estudo do mesmo grupo de pesquisadores (Cong et al., 2011), 28 bebês com IG entre 30 e 32 semanas e de dois a nove dias de idade pós-natal foram analisados em dois grupos: no primeiro, 18 bebês passaram pela posição Canguru de 80 minutos em comparação à posição na incubadora, sem intervenção para a dor, e no segundo, 10 bebês passaram pela posição Canguru de 30 minutos em comparação à posição na incubadora. Para avaliar a dor, os pesquisadores recorreram à análise dos indicadores comportamentais e fisiológicos da PIPP, além do cortisol e soro salivar. Pode-se destacar que tanto os valores obtidos pela PIPP como os dados do cortisol e soro salivar convergiram para um resultado em comum, a diminuição diante de uma intervenção para alívio de dor, a posição Canguru. Na primeira fase do estudo, em que se utilizou do Canguru de 80 minutos, os valores da PIPP não diferiram significativamente entre a posição Canguru e a incubadora. Contudo, na segunda fase do estudo, em que se realizou o Canguru de 30 minutos, os valores da PIPP foram clinicamente menores. Quanto ao cortisol salivar avaliado, este foi significativamente mais baixo na posição Canguru de 30 minutos do que nas duas outras condições. As diminuições dos níveis de cortisol indicaram menos estresse e dor durante a coleta de sangue para o bebê nascido prematuro, quando está em contato pele a pele com a mãe.

Método Canguru como estratégia de alívio de dor realizado por diferentes cuidadores. Nesta categoria, dois estudos tiveram por objetivo analisar a efetividade do Método Canguru com outros cuidadores, diferente das mães. O estudo de Johnston et al. (2011) avaliou 62 bebês nascidos prematuros, com IG de 28 a 36 semanas e que foram submetidos à punção do calcâneo. A comparação deste estudo foi realizada entre a mãe e o pai dos bebês nascidos prematuros, que seguravam os bebês na posição Canguru. Os parâmetros utilizados para observar a dor foram as medidas fisiológicas (frequência cardíaca e saturação do oxigênio) e comportamentais (fronte saliente, os olhos apertados e o sulco nasolabial franzido), utilizando-se como instrumento para essa avaliação, a PIPP. Os resultados obtidos apontaram para uma diferença marginal do ponto de vista estatístico entre a condição de ser a mãe ou o pai, tendo a mãe maior efeito para alívio da dor no recém-nascido do que o pai. Nos segundos após a punção do calcâneo, os valores obtidos pela PIPP foram menores nos bebês que estavam com a mãe, além da frequência cardíaca ter retornado ao mesmo valor da linha de base, na fase de recuperação, em menor tempo com a mãe do que com o pai.

Em outro estudo de Johnston et al. (2012) realizou a comparação entre a posição Canguru com a mãe e com outra pessoa do sexo feminino, escolhida pela mãe do bebê (sogra, cunhada, amigas das mães, outra mãe da unidade ou pro- 
fissional de saúde da unidade), em uma amostra de 18 bebês nascidos prematuros, com IG entre 28 a 36 semanas. Ressalta-se que neste estudo a amostra foi pequena, o que os próprios autores apontaram como uma importante limitação, devido às muitas recusas por parte das mães em aceitar a participar do estudo. A avaliação da dor foi realizada com medidas fisiológicas (frequência cardíaca e saturação do oxigênio) e comportamentais (fronte saliente, os olhos apertados e o sulco nasolabial franzido) por meio da PIPP. Houve a alteração das medidas fisiológicas e dos indicadores comportamentais com a intervenção para o alívio de dor, entretanto, não houve um resultado conclusivo para se afirmar que o contato com a mãe teve efeito maior de alívio a dor na posição Canguru.

Método Canguru vs. Amamentação para o alívio da dor. O estudo de Nanavati et al. (2013) avaliou 50 bebês, com uma média na IG de 32 semanas e com peso ao nascimento de $1.250 \mathrm{~g}$, a fim de comparar a efetividade de dois métodos não-farmacológicos no alívio da dor: o método Canguru e o aleitamento materno. Inicialmente, houve uma primeira fase do estudo, em que foi realizada uma avaliação para comprovar se a remoção de esparadrapo se enquadrava como um procedimento doloroso para bebês nascidos prematuros e de extremo baixo peso, e posteriormente, foi realizada a comparação entre as duas modalidades de alívio de dor. Utilizou-se das medidas fisiológicas do bebê (frequência cardíaca e saturação do oxigênio), assim como dos indicadores comportamentais (atividade corporal do bebê, estado dos olhos abertos ou fechados e movimentos faciais). Verificou-se, na primeira fase do estudo, que a remoção do esparadrapo em bebês nascidos prematuros de extremo baixo peso é um procedimento doloroso, tendo os dados da PIPP colaborado para esta conclusão. Na segunda fase do estudo, os valores obtidos pela PIPP foram significativamente menores após ambas as intervenções, indicando dor leve ou não dor. Os níveis de frequência cardíaca e saturação do oxigênio também apontaram resultados positivos com o uso de ambas as intervenções, mantendo-se estáveis. Portanto, verificou-se que tanto a posição Canguru quanto o aleitamento materno foram efetivos no alívio da dor, entretanto, não foram observadas diferenças significativas entre os grupos.

Método Canguru no alívio de dor e relação com características maternas. Nesta categoria, houve somente um estudo com avaliação tanto do bebê quanto das características maternas. No estudo de Castral et al. (2012) analisou-se 49 bebês com IG menor de 36 semanas, clinicamente estáveis e as mães que tinham capacidade cognitiva para compreender os instrumentos utilizados e não apresentavam diagnóstico psiquiátrico, exceto para depressão e ansiedade. Neste estudo, foi investigada a associação entre os fatores maternos (comportamento desta durante o evento doloroso no bebê, depressão e/ou ansiedade e estresse) e a resposta à dor e ao estresse dos bebês na posição Canguru. Foram observados os indicadores comportamentais (expressões faciais, estado de sono e vigília, choro) e fisiológicos (frequência cardíaca e cortisol salivar) dos bebês, utilizando-se do NFCS para avaliar a dor dos bebês. Para a avaliação dos comportamentos maternos típicos ou tipicamente depressivos, utilizou-se o Maternal Mood and Behavior during her Infant Pain Coding System - MMBIPCS; o Inventário de Depressão de Beck (IDB) e o Inventário de Ansiedade de Beck (IAB) para análise do nível de depressão e ansiedade, respectivamente; e o Structured Clinical Interview for DSM-IV-SCID-CV, para descrever o histórico psiquiátrico das mães, além da coleta do cortisol salivar também da mãe. Foram observadas associações significativas que sugerem uma corregulação entre a mãe e o bebê neste contexto neonatal. Houve associação entre o nível de cortisol materno e diversas das variáveis neonatais analisadas, sendo estas, escores da NFCS, duração do choro, frequência cardíaca e cortisol salivar. Observou-se que o nível de cortisol da mãe da noite anterior tinha uma relação próxima com o nível de frequência cardíaca do bebê ao realizar o contato pele a pele no dia seguinte, havendo uma sugestão de que há uma mediação desta posição Canguru no sistema nervoso autonômico do bebê. Demonstrou também que o comportamento (típico/tipicamente depressivo) e a depressão e/ou ansiedade da mãe não interferiram 
nas respostas de dor e estresse do bebê, além de haver a sugestão de que a realização do contato pele a pele entre mãe e bebê pode ter amenizado os efeitos negativos da depressão e ansiedade das mães.

\section{Discussão}

Os achados da presente revisão sistemática de literatura comprovam a efetividade do contato pele a pele como uma estratégia de manejo não farmacológico para alívio de dor em neonatos pré-termo durante o tratamento intensivo na fase neonatal. Quanto aos delineamentos dos estudos, cinco dos estudos revisados utilizaram-se de um delineamento randomizado cruzado, mostrando rigor metodológico dos estudos. Os estudos clínicos randomizados são considerados o "padrão-ouro" em pesquisa na área clínica e de saúde e que são recomendados para o teste de efetividade e eficácia de intervenções (Greenhalgh, 2013).

Em 11 de 12 estudos, os resultados foram positivos quanto à efetividade desta intervenção para o alívio de dor nos momentos de procedimentos de dor aguda. Apenas em um estudo o resultado foi negativo, porém observou-se que o contato pele a pele não havia sido realizado durante o procedimento invasivo e doloroso, e sim em outros momentos do dia independentemente do momento de dor. Há também a possibilidade de não haver um resultado positivo devido ao fato de que, a pedido dos pais, os bebês que estavam na posição da incubadora também poderiam ser colocados na posição Canguru por 15 minutos diários, não havendo um controle quanto essa variável no estudo. Destaca-se que mesmo os resultados sendo negativos e a metodologia deste artigo se diferenciar dos demais, é importante a inclusão deste na presente revisão sistemática, como forma de certificação de que esta intervenção para a dor é recomendada em associação direta com procedimentos que provocam dor aguda. Vale destacar que os procedimentos dolorosos foram realizados conforme a prescrição da demanda clínica pertinente para cada caso. A intervenção do contato pele a pele foi utilizada quando os bebês estavam clinica- mente estáveis e mediante recomendação e liberação médica, sendo este um importante aspecto a ser observado na utilização deste procedimento para o alívio de dor.

Quanto à duração do contato pele a pele, ressalta-se que os estudos analisaram diversos tempos e que todos apresentaram resultados positivos no alívio da dor, entretanto o mais efetivo foi de 30 minutos. O contato pele a pele com as mães mostrou ser efetivo para alívio de dor e alguns estudos avançaram para comparar a efetividade desta estratégia com outras pessoas, além da mãe. Os achados preliminares mostraram que o contato pele a pele, tanto com as mães quanto com os pais, parece ser eficaz na diminuição da dor dos bebês nascidos prematuros. Porém, os resultados são inconclusivos quando o pele a pele foi realizado por outras mulheres. Houve grande recusa das mães em aceitar participar do estudo que se dedicou a esta questão de comparar a mãe e outras mulheres. As mães tiveram dificuldade de aceitar que outra pessoa do sexo feminino permanecesse em uma condição tão íntima com seu bebê recém-nascido no contato pele a pele. $\mathrm{O}$ estudo destacou que a maioria das mulheres que consentiram sua participação na pesquisa pediu para que a outra pessoa do sexo feminino fosse uma integrante da equipe de enfermagem, podendo-se supor que as mães tinham mais segurança ao ser uma enfermeira treinada e que tinha contato direto com os bebês. Destaca-se que o estudo atendeu ao princípio ético de participação voluntária com termo de consentimento livre e esclarecido assinado. A avaliação dos efeitos do contato pele a pele com outras pessoas, diferentemente das mães, pode ser uma estratégia útil nos casos em que a mãe tem impedimentos ou limitações de estar presente na UTIN ou apresenta quadro clínico grave. Ressalta-se também que nesses estudos, as amostras foram pequenas, o que contribuiu para achados ainda não conclusivos sobre esta questão da efetividade de outras pessoas além da mãe. Novos estudos precisam continuar explorando os efeitos dessa possibilidade de ter o contato pele a pele com pessoas diferentes das mães.

Quanto às formas de se analisar a efetividade desta estratégia, os estudos se utilizaram de 
diversas medidas para avaliar a dor. Pode-se observar que as formas utilizadas foram pertinentes e adequadas, sendo que observou-se uma convergência nos resultados obtidos na maioria dos estudos analisados, em que utilizaram medidas comportamentais e fisiológicas. Destaca-se que para se avaliar a dor adequadamente é necessário a utilização de instrumentos padronizados e validados psicometricamente. A Premature Infant Pain Profile foi a escala mais utilizada, que se trata de uma escala adequada e validada para avaliação de dor em bebês nascidos prematuros. Dos 12 estudos analisados, 10 utilizaram as escalas para medir a dor, com medidas sistemáticas para identificar e verificar os comportamentos relacionados à dor. Em um dos estudos em que não se utilizou uma escala, alguns comportamentos padrões (duração da mímica facial e duração do choro) foram identificados e mediu-se a duração de tempo para avaliar a dor do bebê (Gao et al., 2015). O fato de não usar uma escala padronizada de dor não garantiu que os comportamentos dos bebês fossem específicos de resposta de dor, podendo estes estarem relacionados a estímulos de outra natureza. Outro estudo utilizou-se somente de uma escala para observar o estado comportamental do bebê e suas reações ao estímulo doloroso (Cong, Cusson, Walsh, et al., 2012), tendo assim a observação das reações do bebê à dor, o que limita a especificidade e sensibilidade em relação à avaliação da dor.

Algumas limitações podem ser identificadas nos estudos da presente revisão, que se referem aqueles que não utilizaram instrumentos padronizados e validados para a identificação da dor no bebê prematuro, estudos com amostras pequenas, que não permitem ter resultados conclusivos e dificultam a generalização. Os estudos não tiveram o objetivo de analisar a eficácia do contato pele a pele de acordo com a idade gestacional dos bebês, considerando-se o nível de prematuridade (extremamente prematuros, muito prematuros ou prematuros moderados); a maioria dos estudos usou uma classificação geral da idade gestacional dos bebês. Assim, pode-se pensar em trabalhos futuros em que busquem analisar as diferenças de reações de bebês pre- maturos que nasceram com diferentes idades gestacionais. Recomenda-se também para estudos futuros a utilização dos delineamentos randomizados cruzados, no sentido de fortalecer a literatura com resultados com boa qualidade de evidência científica sobre a intervenção de contato pele a pele. Sugere-se também que as amostras possam ser ampliadas. Além disso, os estudos longitudinais podem avançar em relação aos de corte transversal, a fim de detectar os efeitos a médio prazo no desenvolvimento dos bebês prematuros da intervenção de proteção contra a dor do contato pele a pele.

Com as evidências científicas obtidas até o presente momento, pode-se afirmar a eficácia do contato pele a pele entre mães e recém-nascidos para alívio de dor no contexto hospitalar. Observa-se a necessidade da transferência de conhecimentos científicos do contato pele a pele para a prática, como previamente preconizado pelo Ministério da Saúde (2011). Entretanto, existem os desafios de treinamento de profissionais e a implementação e manutenção de tais protocolos de alívio de dor em hospitais. Portanto, há a necessidade de que essa técnica torne-se rotina clínica, por tratar-se de uma estratégia de baixo custo, além de trazer diversos benefícios para a díade mãe-bebê, promovendo o vínculo e um cuidado à saúde de forma mais sensível e humanizada.

Quanto às limitações da presente revisão, pode-se citar a restrição do critério temporal e a dos idiomas estabelecidos previamente, assim como os artigos obtidos, tendo a limitação de se conseguir gratuitamente os estudos. Outra limitação foi que não houve restrição quanto aos delineamentos dos estudos, tendo-se artigos não randomizados controlados também incluídos. Não se realizou também a aplicação de técnicas estatísticas, usadas em revisões de meta-análise, esta podendo ser uma questão para estudos futuros.

\section{Referências}

Associação Internacional para o Estudo da Dor. (2017). IASP Terminology. (Reprinted and updated from Classification of Chronic Pain: IASP Task Force on Taxonomy, Pt. III, pp. 209-214, by H. Merskey \& N. Bogduk, Eds., 1994, Seat- 
tle, WA: International Association for the Study of Pain Press). Retrieved from http://www.iasppain.org/Education/Content.aspx?ItemNumber $=1698 \&$ navItem Number $=576$

Barker, D. P., \& Rutter, N. (1995). Exposure to invasive procedures in neonatal intensive care unit admissions. Archives of Disease in Childhood. Fetal and Neonatal Edition, 72, 47-48. doi: https://doi.org/10.1136/fn.72.1.F47

Cassiano, R. G. M., Gaspardo, C. M., \& Linhares, M. B. M. (2016). Prematurity, neonatal health status, and later child behavioral/emotional problems: A systematic review. Infant Mental Health Journal, 37(3), 274-288. doi: https://doi. org/10.1002/imhj.21563

Castral, T. C., Warnock, F. F., Ribeiro, L. M., Vasconcelos, M. G. L., Leite, A. M., \& Scochi, C. G. S. (2012). Maternal factors regulating preterm infants' responses to pain and stress while in maternal kangaroo care. Revista Latino-Americana de Enfermagem, 20(3), 435-443. Retrieved from http://www.ncbi.nlm.nih.gov/ pubmed/22991104

Chabra, S. (2013). Subsets of preterm and term infants: Call for consistency in terminology. Developmental Medicine and Child Neurology, 55(7), 673. doi: https://doi.org/10.1111/ dmen. 12126

Charpak, N., Ruiz, J. G., Zupan, J., Cattaneo, A., Figueroa, Z., Tessier, R., ...Worku, B. (2005). Kangaroo Mother Care: 25 years after. Acta Paediatrica (Oslo, Norway), 94(5), 514-522. doi: https://doi.org/10.1080/08035250510027381

Chidambaram, A. G., Manjula, S., Adhisivam, B., \& Bhat, B. V. (2014). Effect of Kangaroo mother care in reducing pain due to heel prick among preterm neonates: A crossover trial. The Journal of Maternal-Fetal \& Neonatal Medicine, 27(5), 488-490. doi: https://doi.org/10.3109/14767058. 2013.818974

Cignacco, E., Hamers, J. P. H., Stoffel, L., van Lingen, R. A., Gessler, P., McDougall, J., \& Nelle, M. (2007). The efficacy of non-pharmacological interventions in the management of procedural pain in preterm and term neonates. A systematic literature review. European Journal of Pain, 11, 139-152. doi: https://doi.org/10.1016/j. ejpain.2006.02.010

Cong, X., Cusson, R. M., Hussain, N., Zhang, D., \& Kelly, S. P. (2012). Kangaroo care and behavior- al and physiologic pain responses in very-lowbirth-weight twins: A case study. Pain Management Nursing, 13(3), 127-138. doi: https://doi. org/10.1016/j.pmn.2010.10.035

Cong, X., Cusson, R. M., Walsh, S., Hussain, N., Ludington-Hoe, S. M., \& Zhang, D. (2012). Effects of Skin-to-skin contact on autonomic pain responses in preterm infants. The Journal of Pain, 13(7), 636-645. doi: https://doi.org/10.1016/j. jpain.2012.02.008

Cong, X., Ludington-Hoe, S. M., \& Walsh, S. (2011). Randomized crossover trial of kangaroo care to reduce biobehavioral pain responses in preterm infants: A pilot study. Biological Research For Nursing, 13(2), 204-216. doi: https://doi. org/10.1177/1099800410385839

Gao, H., Xu, G., Gao, H., Dong, R., Fu, H., Wang, D., ...Zhang, H. (2015). Effect of repeated Kangaroo Mother Care on repeated procedural pain in preterm infants: A randomized controlled trial. International Journal of Nursing Studies, 52(7), 1157-1165. doi: https://doi.org/10.1016/j. ijnurstu.2015.04.006

Greenhalgh, T. (2013). Chegando ao ponto: Do que trata este artigo? In Como ler artigos cientificos: Fundamentos da medicina baseada em evidências (pp. 50-66). Porto Alegre, RS: Artmed.

Grunau, R. V. E. (2002). Early pain in preterm infants. A model of long-term effects A model of long-term effects. Clinics in Perinatology, 29, 373-394.

Johnston, C., Byron, J., Filion, F., Campbell-Yeo, M., Gibbins, S., \& Ng, E. (2012). Alternative female kangaroo care for procedural pain in preterm neonates: A pilot study. Acta Paediatrica, 101(11), 1147-1150. doi: https://doi.org/10.1111/j.16512227.2012.02813.x

Johnston, C., Campbell-Yeo, M., Fernandes, A., Inglis, D., Streiner, D., \& Zee, R. (2014). Skinto-skin care for procedural pain in neonates. In C. Johnston (Ed.), Cochrane Database of Systematic Reviews (Vol. 1, pp. 1-82). Chichester, UK: John Wiley \& Sons. doi: https://doi. org/10.1002/14651858.CD008435.pub2

Johnston, C., Campbell-Yeo, M., \& Filion, F. (2011). Paternal vs maternal kangaroo care for procedural pain in preterm neonates: A randomized crossover trial. Archives of Pediatrics \& Adolescent Medicine, 165(9), 792-796. doi: https://doi. org/10.1001/archpediatrics.2011.130 
Klein, V. C., Gaspardo, C. M., \& Linhares, M. B. M. (2006). Dor, Autorregulação e temperamento em recém-nascidos pré-termo de alto risco. Psicologia: Reflexão e Crítica, 24(3), 504-512. doi: https://doi.org/10.1590/S010279722011000300011

Liberati, A., Altman, D. G., Tetzlaff, J., Mulrow, C., Gøtzsche, P. C., Ioannidis, J. P. A., ...Moher, D. (2009). The PRISMA Statement for Reporting Systematic Reviews and meta-analyses of studies that evaluate health care interventions: Explanation and elaboration. PLoS Medicine, 6(7), e1000100. doi: https://doi.org/10.1371/journal. pmed. 1000100

Linhares, M. B. M. (2016). Estresse precoce no desenvolvimento: Impactos na saúde e mecanismos de proteção. Estudos de Psicologia (Campinas), 33(4), 587-599. doi: https://doi. org/10.1590/1982-02752016000400003

Linhares, M. B. M., \& Doca, F. (2010). Dor em neonatos e crianças: Avaliação e intervenções não farmacológicas. Temas em Psicologia, 18(2), 307-325. Recuperado em http:// pepsic.bvsalud.org/scielo.php?pid $=\mathrm{S} 1413$ $-389 \times 2010000200006 \& \mathrm{script}=\mathrm{sci}$ arttext\&tlng $=$ en

Ministério da Saúde. (2011). Dor no recém-nascido. In Atenção à Saúde do Recém-Nascido: Guia para os profissionais de saúde. Intervenções comuns, icterícia e infecções: Vol. 2 (pp. 33-45). Brasília, DF: Autor.

Ministério da Saúde. (2015). Nascidos Vivos - Brasil. Recuperado em http://tabnet.datasus.gov.br/cgi/ tabcgi.exe?sinasc/cnv/nvuf.def

Mitchell, A. J., Yates, C. C., Williams, D. K., Chang, J. Y., \& Hall, R. W. (2013). Does daily kangaroo care provide sustained pain and stress relief in preterm infants? Journal of NeonatalPerinatal Medicine, 6(1), 45-52. doi: https://doi. org/10.3233/NPM-1364212

Nanavati, R. N., Balan, R., \& Kabra, N. S. (2013). Effect of kangaroo mother care vs expressed breast milk administration on pain associated with re- moval of adhesive tape in very low birth weight neonates: A randomized controlled trial. Indian Pediatrics, 50, 1011-1015. Retrieved from http://www.indianpediatrics.net/nov2013/1011. pdf

Nimbalkar, S. M., Chaudhary, N. S., Gadhavi, K. V., \& Phatak, A. (2013). Kangaroo mother care in reducing pain in preterm neonates on heel prick. Indian Journal of Pediatrics, 80(1), 6-10. doi: https://doi.org/10.1007/s12098-012-0760-6

Olsson, E., Ahlsén, G., \& Eriksson, M. (2016). Skinto-skin contact reduces near-infrared spectroscopy pain responses in premature infants during blood sampling. Acta Paediatrica, 105(4), 376380. doi: https://doi.org/10.1111/apa.13180

Pereira, F. L., Goes, F. D. S. N. de, Fonseca, L. M. M., Scochi, C. G. S., Castral, T. C., \& Leite, A. M. (2013). A manipulação de prematuros em uma Unidade de Terapia Intensiva Neonatal. Revista da Escola de Enfermagem da USP, 47(6), 1272-1278. doi: https://doi.org/10.1590/S0080623420130000600003

Valeri, B. O., Holsti, L., \& Linhares, M. B. M. (2015). Neonatal pain and developmental outcomes in children born preterm: A systematic review. The Clinical Journal of Pain, 31(4), 355-362. doi: https://doi.org/10.1097/ AJP.0000000000000114

Warnock, F. F., Castral, T. C., Brant, R., Sekilian, M., Leite, A. M., Owens, S. de la P., \& Scochi, C. G. S. (2010). Brief report: Maternal kangaroo care for neonatal pain relief: A systematic narrative review. Journal of Pediatric Psychology, 35(9), 975-984. doi: https://doi.org/10.1093/jpepsy/ jsp 123

World Health Organization. (2016). Preterm birth. Retrieved from http://www.who.int/mediacentre/factsheets/fs363/en/

Recebido: 22/08/2017

$1^{a}$ revisão: 04/10/2017

Aceite final: $18 / 10 / 2017$

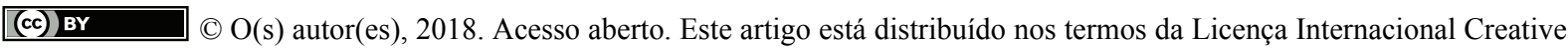
Commons Atribuição 4.0 (http://creativecommons.org/licenses/by/4.0/), que permite o uso, distribuição e reprodução sem restrições em qualquer meio, desde que você dê crédito apropriado ao(s) autor(es) original(ais) e à fonte, fornecer um link para a licença Creative Commons e indicar se as alterações foram feitas. 